\title{
A two-dimensional computational model of turbulent atmospheric surface flows with drifting snow
}

\author{
G. E. LISTON, \\ Climate and Radiation Branch, NASA/Goddard Space Flight Center, Greenbelt, MD 20771, U.S.A. \\ R. L. BROWN AND J. D. Dent \\ Department of Civil and Agricultural Engineering, Montana State University, Bozeman, MT 59717, U.S.A.
}

\begin{abstract}
A physically based computational model of drifting snow in twodimensional terrain is developed. The model considers the case where wind speeds are low enough to neglect the transport of particles from the saltation layer into the turbulent flow field. The model has two distinct parts, one describing the turbulent airflow, and a second describing the mass-transport process and resulting snowaccumulation patterns produced by saltation transport. The turbulent-flow model consists of a general solution of the time-averaged, two-dimensional Navier-Stokes equations, where the $k-\epsilon$ turbulence model is used to close the system of equations. The turbulent-flow model is coupled to a saltation model to compute the time evolution of the surface wind fields and snowdrift formation in the vicinity of a solid fence. Modeled wind fields and snow-accumulation profiles are similar to published field and experimental data.
\end{abstract}

\section{INTRODUCTION}

Outside our doors in the winter environment, whether it be in the city, the country, or the mountains, snow depth is found to be highly variable. The dominant process which produces these spatially varying snow depths is the redistribution of snow by wind. In blowing and drifting snow, two distinct flow regimes are found. At the surface is the saltation layer which is characterized by particles repeatedly impacting the surface, dislodging additional particles into the air to be brought back to the surface under the influence of gravity. Above this layer, the flow can be described as a turbulent, two-phase mixture of air and snow particles. These two layers are highly coupled, and therefore an accurate simulation of snow transport relies heavily on the descriptions of motions contained within both of these layers. Recently, computational and physical modeling efforts have attempted to describe the physical processes associated with snow transport. Decker and Brown (1985) utilized modern mixture theory to study blowing snow in mountainous terrain. Uematsu and others (1989) developed a two-dimensional finiteelement model of snowdrift development and Uematsu and others (1991) modeled snowdrift around threedimensional obstructions. We present a computational model which describes the turbulent flow of air above the saltation layer. This flow field is coupled, through their common boundary, to a saltation model. The coupled model is applied to the case of saltation-dominated mass transport, and is used to simulate snow-accumulation patterns in the region of a solid vertical fence.

\section{GOVERNING EQUATIONS}

\section{Turbulence model}

Based on the universal conservation laws of mass and momentum, the governing equations for a Newtonian fluid are commonly referred to as the Navier-Stokes equations. Reynolds, or time averaging, introduces additional terms which must be modeled to obtain a closed system of equations. Restricting our interest to steady, two-dimensional flows, and adopting the concept of a turbulent-viscosity leads to the following continuity, and $x$ and $z$-momentum equations:

$$
\begin{aligned}
\frac{\partial u}{\partial x}+\frac{\partial w}{\partial z} & =0 \\
u \frac{\partial u}{\partial x}+w \frac{\partial u}{\partial z} & =-\frac{1}{\rho} \frac{\partial p}{\partial x}+\frac{\partial}{\partial x}\left(\nu_{\mathrm{t}} \frac{\partial u}{\partial x}\right)+\frac{\partial}{\partial z}\left(\nu_{\mathrm{t}} \frac{\partial u}{\partial z}\right) \\
u \frac{\partial w}{\partial x}+w \frac{\partial w}{\partial z} & =-\frac{1}{\rho} \frac{\partial p}{\partial z}+\frac{\partial}{\partial x}\left(\nu_{\mathrm{t}} \frac{\partial w}{\partial x}\right)+\frac{\partial}{\partial z}\left(\nu_{\mathrm{t}} \frac{\partial w}{\partial z}\right)
\end{aligned}
$$

where $u$ and $w$ are the mean velocity components in the $x$ (horizontal) and $z$ (vertical) directions, respectively; $p$ is pressure; $\rho$ is the fluid density; and $\nu_{\mathrm{t}}$ is the turbulentviscosity coefficient which is a property of the flow and may vary greatly over the flow field.

To close this system of equations, $\nu_{\mathrm{t}}$ must be formulated in terms of the mean flow variables. Existing turbulence models range from simple models based on Prandtl's mixing-length hypothesis, to those that use differential transport equations for the individual turbu- 
lent stresses and fluxes. The simple models based on the Prandtl mixing-length hypothesis suffer from several drawbacks. One primary failing is that the hypothesis implies that the turbulence is in local equilibrium, consequently the model is unable to account for the transport and history effects of turbulence. In addition, since it is difficult to prescribe the mixing-length distribution in any but the simplest flows, these models are not applicable to more complex, separating flows (Rodi, 1980). A promising alternative is the $k-\epsilon$ turbulence model of Jones and Launder (1972). This is a two-equation model which uses partial differential transport equations to describe the distribution of turbulent kinetic energy, $k$, and the dissipation rate, $\epsilon$, of the turbulent kinetic energy.

Since $\nu_{\mathrm{t}}$ is proportional to $k^{2} / \epsilon$, it is assumed that

$$
\nu_{\mathrm{t}}=c_{\mu} \frac{k^{2}}{\epsilon}
$$

where $c_{\mu}$ is a constant. For the flow of interest here, the turbulent kinetic energy transport equation is

$$
u \frac{\partial k}{\partial x}+w \frac{\partial k}{\partial z}=\frac{\partial}{\partial x}\left(\frac{\nu_{\mathrm{t}}}{\sigma_{k}} \cdot \frac{\partial k}{\partial x}\right)+\frac{\partial}{\partial z}\left(\frac{\nu_{\mathrm{t}}}{\sigma_{k}} \cdot \frac{\partial k}{\partial z}\right)
$$

$$
+\nu_{\mathrm{t}}\left(\frac{\partial u}{\partial z}-\frac{\partial w}{\partial x}\right)^{2}-\epsilon
$$

where term (i) describes convective transport of $k$, term (ii) is the diffusive transport, term (iii) describes the production of $k$ through shear, and term (iv) is the rate of viscous dissipation. The $\epsilon$ equation is

$$
\begin{aligned}
u \frac{\partial \epsilon}{\partial x}+w \frac{\partial \epsilon}{\partial z}= & \frac{\partial}{\partial x}\left(\frac{\nu_{\mathrm{t}}}{\sigma_{\epsilon}} \cdot \frac{\partial \epsilon}{\partial x}\right)+\frac{\partial}{\partial z}\left(\frac{\nu_{\mathrm{t}}}{\sigma_{\epsilon}} \cdot \frac{\partial \epsilon}{\partial z}\right) \\
& +c_{1 \epsilon} \frac{\epsilon}{k} \nu_{\mathrm{t}}\left(\frac{\partial u}{\partial z}-\frac{\partial w}{\partial x}\right)^{2}-c_{2 \epsilon} \frac{\epsilon^{2}}{k}
\end{aligned}
$$

where each term has a meaning similar to those found in the $k$ equation. Based on the work of Jones and Launder (1972) the empirical constants in these equations are: $c_{\mu}=0.09, c_{1 \epsilon}=1.44, c_{2 \epsilon}=1.92, \sigma_{k}=1.0, \sigma_{\epsilon}=1.3$. For atmospheric flows, as opposed to engineering or hydraulic flows, the values of $c_{\mu}=0.03$ and $c_{1 \epsilon}=1.16$ have been suggested (Sutton and others, 1986). Since the mid-1970s the $k-\epsilon$ model has been tested extensively and has found success for a variety of different flows, including free shear flows, wall boundary layers, and flows involving recirculation.

\section{Boundary conditions}

To solve the governing equations, boundary conditions must be applied to all boundaries of the computational domain for the dependent variables, $u, w, p, k$ and $\epsilon$. Representative atmospheric profiles of velocity, turbulent kinetic energy, and dissipation rate are required for the inflow boundary conditions. Wind profiles within a neutrally stratified boundary layer can be approximated by

$$
u=\frac{u_{*}}{\kappa} \ln \left(z / z_{0}\right)
$$

where $u_{*}$ is the shear or friction velocity, $z$ is the height above the surface, $z_{0}$ is the roughness length, and $\kappa=0.4$ is von Karman's constant. The rate of dissipation above the viscous sublayer is given by

$$
\epsilon=\frac{u_{*}^{3}}{\kappa z},
$$

(Sorbjan, 1989). Frost and others (1975) provided atmospheric profiles of turbulent kinetic energy $k$ for the same conditions, where

$$
k=\frac{u_{*}^{2}}{\sqrt{c_{\mu}}} .
$$

Boundary conditions for the solid surfaces are formulated by choosing a computational domain which is slightly removed from the solid boundary, and then applying semi-empirical wall functions to the gap between the solid boundary and computational boundary. The logarithmic profile given by Equation (7) is used as the $u$ velocity boundary condition on all horizontal solid surfaces including the top of the fence and on the tops of snowdrifts. In the presence of saltation, Owen (1964) suggested that the effective roughness length of the saltating surface is proportional to $u_{*}^{2} / 2 g$, where $g$ is the gravitational acceleration. In light of the saltation modeling assumptions imposed in the current study, as discussed below, the implementation of a non-linear relationship between $u$ and $u_{*}$ does not appear justified. A complete summary of the boundary conditions applied to this problem is found in Table 1. Combining Equations (1)-(6) and the boundary conditions yields the coupled, highly non-linear system of equations which models the steady-state, two-dimensional turbulent flow field.

\section{Saltation model}

The presence of saltation is dependent upon the relative magnitudes of the shear strength of the snow surface and the shear stress at the surface produced by the wind above. Experimental studies have found threshold shearvelocity values ranging from $<0.1 \mathrm{~m} \mathrm{~s}^{-1}$ for light, dry snow, to $>0.4 \mathrm{~m} \mathrm{~s}^{-1}$ for old, hardened snow. Under moderate wind speeds, measurements above a horizontal surface show a very rapid decrease in mass flux with height through the saltation layer. Kind (1981) reported that, for a wind speed of $10 \mathrm{~m} \mathrm{~s}^{-1}$, approximately $90 \%$ of the total saltating mass flux was contained within the first $3 \mathrm{~cm}$ above the surface. At higher wind speeds the suspension of particles in the turbulent-flow regime plays a significant role in the mass transport (Kind, 1992), and is strongly dependent upon the threshold shear velocity (Pomeroy and Gray, 1990).

Saltation models have been developed which describe the steady-state transport of saltating snow over uniform horizontal surfaces. For example, Pomeroy and Gray (1990) suggested

$$
Q_{\mathrm{s}}=\frac{0.68 \rho}{g}\left(\frac{u_{* \mathrm{t}}}{u_{*}}\right)\left(u_{*}^{2}-u_{* \mathrm{t}}^{2}\right),
$$

where $Q_{\mathrm{s}}$ is the total mass-transport rate per unit lateral dimension, $u_{* t}$ is the threshold shear or friction velocity, and 0.68 is an empirical constant. 
Table 1. Boundary conditions used in the computational model, where $\mathrm{n}$ is the direction perpendicular to the boundary
Inflow boundary
Upper boundary
Outflow boundary
Horizontal surfaces
Vertical surfaces

$\begin{array}{ccclcc}u & \begin{array}{c}\text { Equation } \\ (7)\end{array} & \frac{\partial u}{\partial \mathrm{n}}=0 & \frac{\partial u}{\partial \mathrm{n}}=0 & \begin{array}{c}\text { Equation } \\ (7)\end{array} & 0 \\ w & \frac{\partial w}{\partial \mathrm{n}}=0 & \frac{\partial w}{\partial \mathrm{n}}=0 & 0 & 0 \\ p & \frac{\partial p}{\partial \mathrm{n}}=0 & \frac{\partial p}{\partial \mathrm{n}}=0 & \frac{\partial p}{\partial \mathrm{n}}=0 & \frac{\partial p}{\partial \mathrm{n}}=0 \\ k & \frac{\partial p}{\partial \mathrm{n}}=0 & \frac{\partial k}{\partial \mathrm{n}}=0 & \frac{\partial k}{\partial \mathrm{n}}=0 & \begin{array}{c}\text { Equation } \\ (9)\end{array} & \frac{\partial k}{\partial \mathrm{n}}=0 \\ \epsilon & \begin{array}{c}\text { Equation } \\ (9)\end{array} & \frac{\partial \epsilon}{\partial \mathrm{n}}=0 & \frac{\partial \epsilon}{\partial \mathrm{n}}=0 & \begin{array}{c}\text { Equation } \\ (8)\end{array} & \frac{\partial \epsilon}{\partial \mathrm{n}}=0\end{array}$

The saltation model implies that decreasing shear velocity leads to decreasing transport, and that when the shear velocity drops below the threshold velocity, mass transport will cease. Since Equation (10) was developed assuming a fetch long enough to provide a steady-state supply of particles, it is not directly applicable to the case of two-dimensional flow where the surface shear velocity can vary significantly over short distances. For example, consider the increase in shear velocity as the wind travels over a small bump: while the saltation model implies an
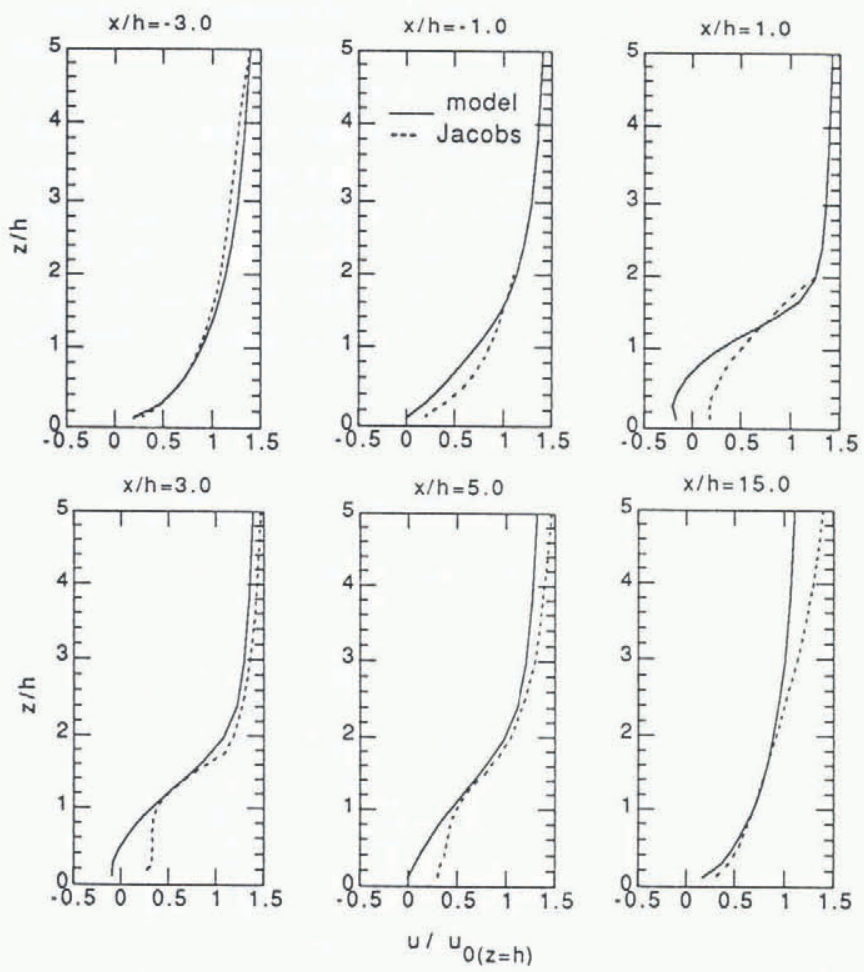

Fig. 1. Comparison of velocity profiles measured by Jacobs (1984) and computed by the model. increase in mass transport, that mass is not necessarily available. To apply the saltation model to the twodimensional flow field of the present study, only the switching feature of the model is used, thus it only determines the presence or lack of transport. It is assumed that saltation ceases and snow accumulates at the location where the shear velocity drops below the threshold. In this simplified form, transport rates and the time required for drift traps to reach equilibrium cannot be computed.

The turbulent flow model is solved by discretizing the flow domain into an array of rectangular grid boxes. The surface shear velocity, $u_{*}$, is computed through the turbulent flow field calculation, and is then used to run the saltation model upon providing a threshold shear velocity, $u_{* t}$. Snow-accumulation patterns are developed by assuming that snow will be deposited within the surface grid box where the shear velocity first drops below the threshold. When enough snow accumulates to fill that grid box, it is blocked out and a new lower-boundary configuration results. The flow field is then recomputed and the process is repeated until an equilibrium drift has been formed which contains no regions of shear velocity below the threshold.

\section{COMPUTATIONAL RESULTS AND DISCUSSION}

\section{Flow over a solid fence}

Two experimental studies of atmospheric flow over a long, thin solid fence are used to verify the turbulent-flow model output. The first study is a full-scale field experiment (Jacobs, 1984), while the second study was conducted in an atmospheric wind tunnel (Perera, 1981). In Jacobs' experiment, wind-flow profiles were measured at nine locations aligned perpendicular to a thin solid fence of $2 \mathrm{~m}$ height, $64 \mathrm{~m}$ length and $0.02 \mathrm{~m}$ thickness. The 

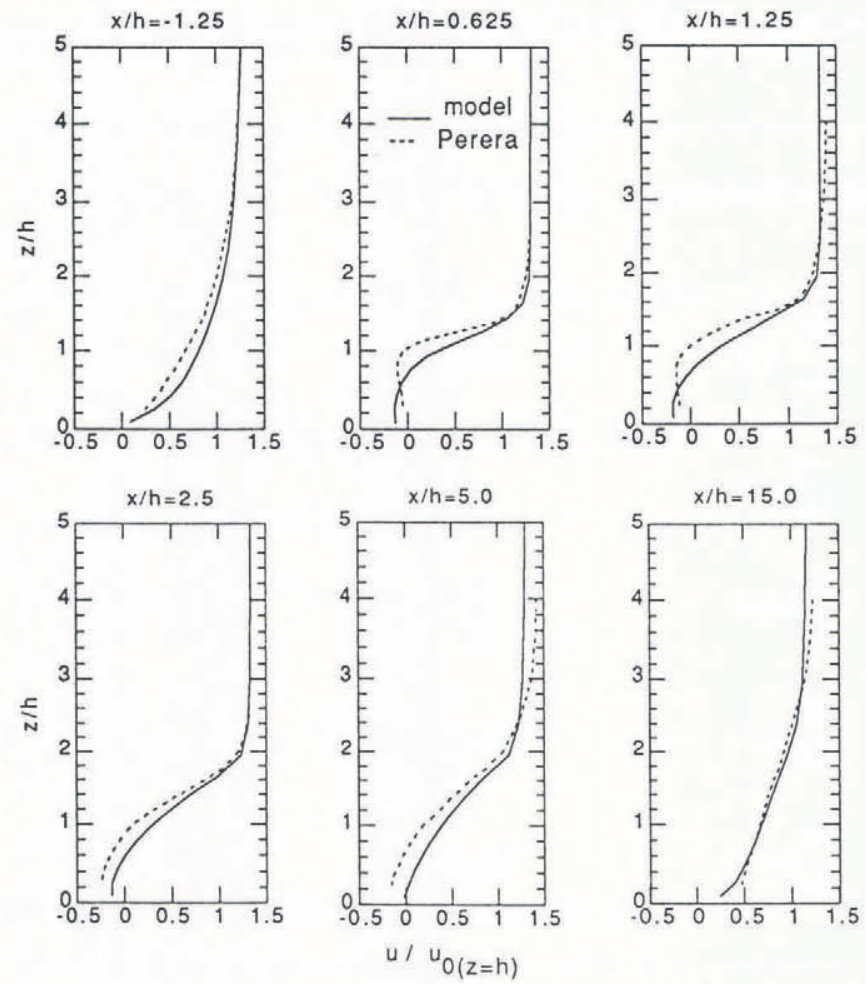

Fig. 2. Comparison of velocity profiles measured by Perera (1981) and computed by the model.

surface cover consisted of mowed heather with roughness length of $0.035 \mathrm{~m}$. Wind speeds were measured using cup anemometers.

Duplicating this experiment with the computational model shows that all major features of the flow have been simulated. Jacobs found a recirculation eddy at the front of the fence, starting near the surface at about $-0.5 h$, where $h$ is the fence height, and reattaching on the fence at a height between $0.5 h$ and $1.0 h$. The model places the eddy at approximately $-0.8 h$ for the surface and $0.6 h$ for the fence. Behind the fence Jacobs found a recirculation eddy which started at the top of the fence and extended a distance of between $5 h$ and $10 h$ to the ground surface. The model produced a reattachment point a distance $5.8 h$ from the fence. Figure 1 shows a comparison of the velocity fields measured by Jacobs and computed by the model. Displayed are the velocity profiles measured at locations $x / h=-3.0,-1.0,1.0,3.0,5.0$ and 15.0, where the velocity has been scaled by the incoming velocity at fence height $h$. The model produces a realistic depiction of the measured velocity field. The largest discrepancies occur in the lee recirculation eddy where, although identified visually in Jacobs' study, the cup anemometers have not resolved the flow direction. Further inaccuracies in field measurement techniques and variations in such features as surface roughness and wind-approach direction do not warrant a more detailed analysis of the velocity differences.

Perera's experiment was also a two-dimensional, thin fence study, but here the roughness length was significantly smaller at $0.00036 \mathrm{~m}$. Wind speeds were measured in a wind tunnel using pulsed-wire anemometers. These anemometers, unlike cup and hot-wire anemometers, have the ability to distinguish between positive and negative directions of wind flow. The computational reproduction of this study is visually similar to the Jacobs flow field: all major features of the flow have been reproduced. The smaller roughness length has extended the windward recirculation eddy to $-1.1 h$ at the surface, and reduced the lee eddy to $5.5 \mathrm{~h}$. Figure 2 compares the experimental and model velocity profiles at locations $x / h=-1.25,0.625,1.25,2.5,5.0$ and 15.0 for Perera's study.

\section{Snow accumulation patterns}

The two-dimensional flow over a solid vertical fence is used as a test case for the coupled flow-saltation model. The fence is $2 \mathrm{~m}$ high and $0.5 \mathrm{~m}$ thick, and the incoming velocity profile is defined by Equation (7) with a $10 \mathrm{~m}$ velocity of $10 \mathrm{~m} \mathrm{~s}^{-1}$ and a roughness length of $0.001 \mathrm{~m}$. The computed flow field for this boundary configuration is given in Figure 3.

The equilibrium drift configuration computed for a threshold shear velocity $u_{* \mathrm{t}}=0.2 \mathrm{~m} \mathrm{~s}^{-1}$ is given in Figure 4. The evolution of the profile is illustrated in Figure 5, where the numbers indicate the sequence in which grid boxes were blocked out as the drift evolved. Figure 5 shows the drift first forming windward of the fence, in the region of the recirculation eddy, where a shear velocity below the threshold is first encountered. This drift builds at a position removed from the fence and then fills in between the fence and the drift. After the windward drift reaches equilibrium, snow begins to accumulate in the lee

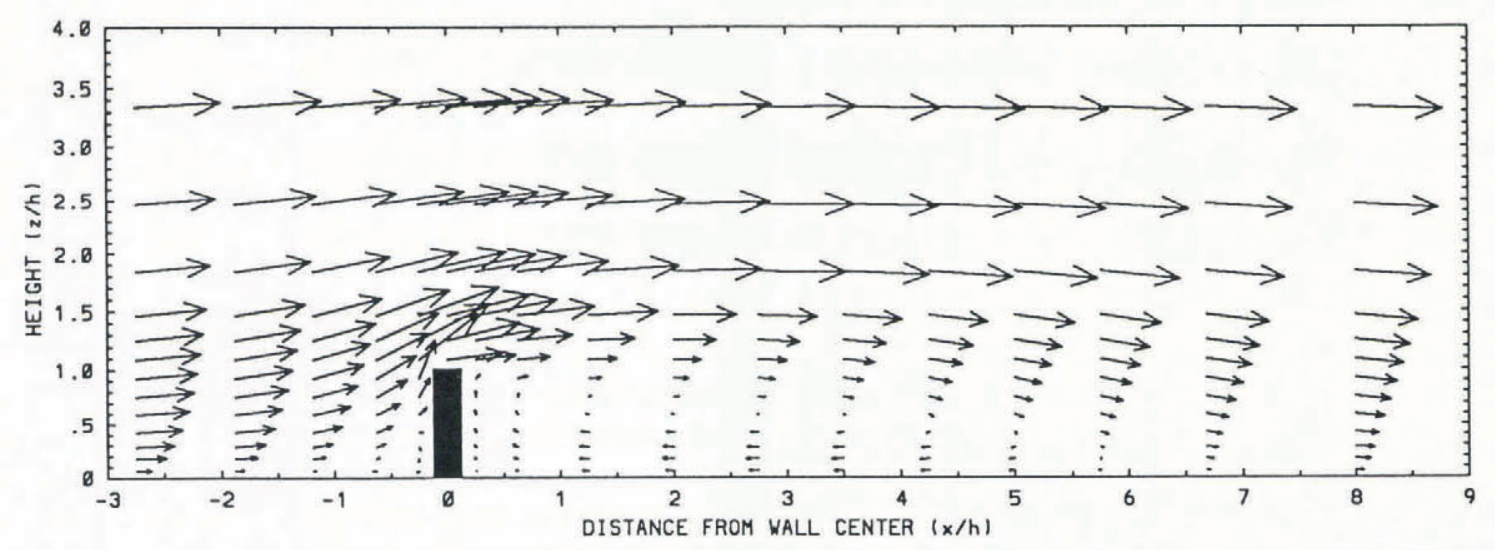

Fig. 3. Computed flow field for test-case problem of single-phase, turbulent flow over a solid fence. 


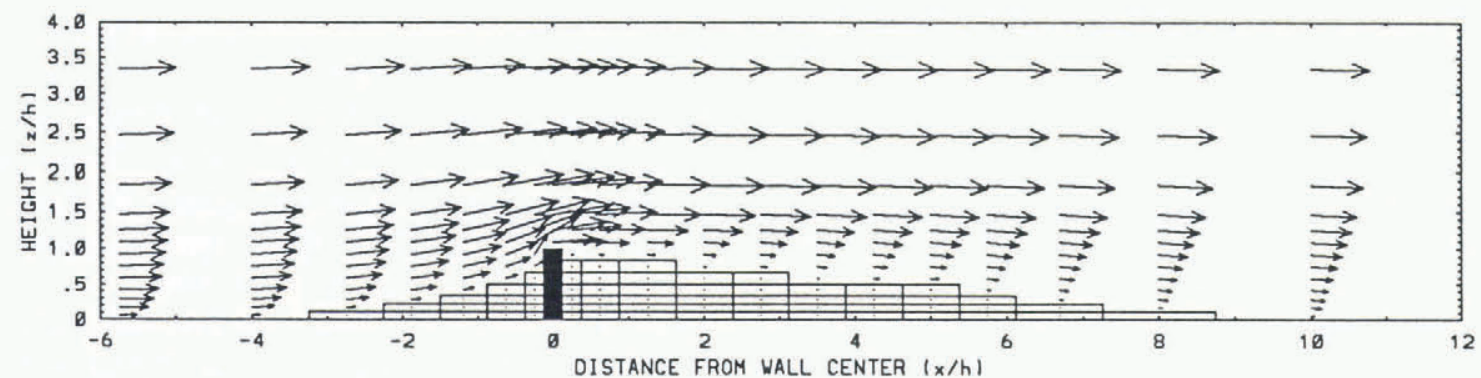

Fig. 4. Equilibrium snowdrift and flow-field configuration computed for a threshold shear velocity of $u_{* \mathrm{t}}=0.2 \mathrm{~ms}^{-1}$ for the test-case problem of single-phase, turbulent flow over a solid fence.

of the fence. The shoulder or scarp formation found in the lee drift at intermediate stages, for example the one present after the 41st blocked grid box, is a commonly observed feature of snowdrift formation (Mellor, 1965; Tabler, 1975). As the lee drift extends downwind of the fence, the flow field is modified and may produce additional accumulation at locations windward of the main drift scarp. This is illustrated, for example, by the blocking of the 51st grid box. This result also occurs with the drift windward of the fence. Increasing (or decreasing) the threshold shear velocity will increase (or decrease) the size of the equilibrium drift.

The lee equilibrium drift surface has a slope of 10$13 \%$. For the case of embankment slopes descending from a level plain, Finney (1939) found that no snow accumulates on embankment slopes of less than $17 \%$. Berg and Caine (1975) and Tabler (1975) found only small accumulations on slopes of $17 \%$ for this same $0 \%$ approach-slope configuration. The relatively steep approach slope described by the drift windward of the fence in the computational experiment produces a longer drift than those produced in the above examples which have approach slopes of zero. This is consistent with Tabler's study where, for an approach slope of approximately $10 \%$, snow accumulated on a lee slope of approximately $10 \%$. Planned model improvements which include accounting for the transport of snow due to turbulent suspension and/or precipitation are expected to aid in filling down-wind regions and help create snow-accumulation sequences which more closely fit natural accumulation patterns.

\section{SUMMARY AND GONCLUSIONS}

A physically based computational model describing turbulent flow has been developed and applied to the problem of drifting snow. The modeled flow is composed of two distinct layers, one containing an upper turbulent flow of air, and a second containing the snow transported by saltation very close to the ground. The coupled model addresses the case where wind speeds are low enough to neglect the transport of particles from the saltation layer into the turbulent flow field. As an initial approximation we have assumed that an appropriate foundation for modeling wind-transported snows in complex landscapes is an accurate representation of the turbulent flow field. This is then used to drive a saltation model. In nature, a two-way coupling exists between the layers; each affecting the behavior of the other. Future model improvements will include relaxing the one-way coupling between the two models and the consideration of particulate transport within the turbulent flow field.

\section{ACKNOWLEDGEMENTS}

The authors wish to express their sincere gratitude to Drs Bowers, Demetriades, Hansen and Vogel for their comments and consultation during this modeling effort. This work was supported by the Center for HighElevation Studies, the Department of Civil and Agricultural Engineering, and the Engineering Experiment Station at Montana State University.

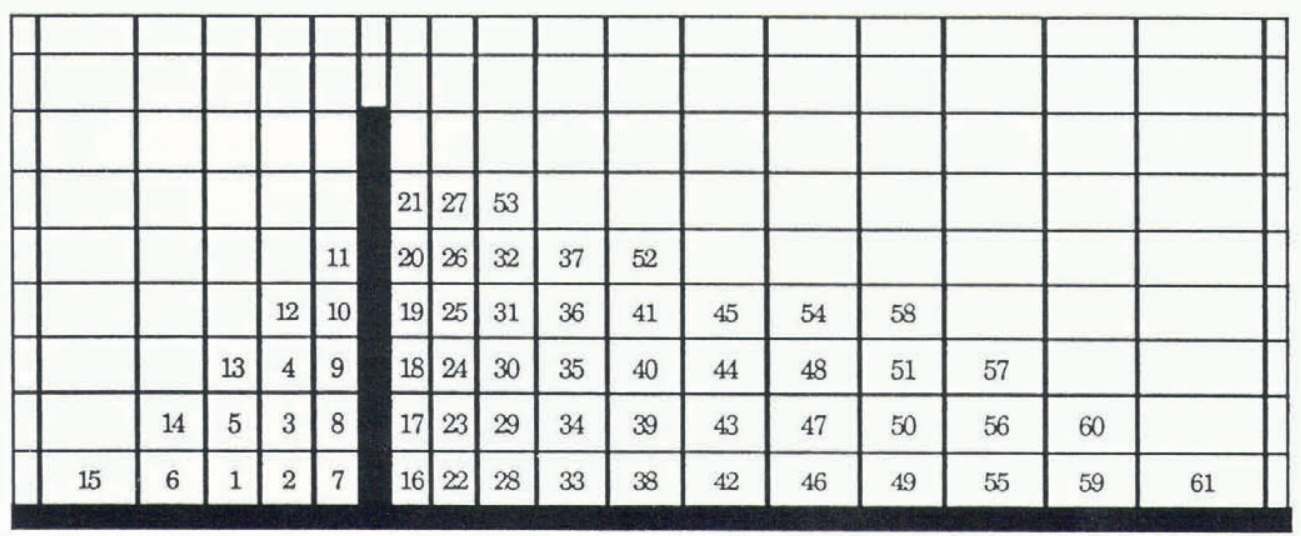

Fig. 5. Progression of snowdrift development for the case $u_{* \mathrm{t}}=0.2 \mathrm{~m} \mathrm{~s}^{-1}$. The numbers indicate the sequence in which the model blocked grid boxes as the drift evolved towards the equilibrium configuration. 


\section{REFERENCES}

Berg, N.H. and N. Caine. 1975. Prediction of natural snowdrift accumulation in alpine areas. Final report to Rocky Mountain Forest and Range Experiment Station. Boulder, CO, University of Colorado. Department of Geography. (USFS 19-388-CA.)

Decker, R. and R.L. Brown. 1985. Two dimensional solutions for a turbulent continuum theory for the atmospheric mixture of snow and air. Ann. Glaciol., 6, 53-58.

Finney, E. A. 1939. Snow drift control by highway design. Michigan State College Engineering Experiment Station. Bulletin 86.

Frost, W., W. L. Harper and G. H. Fichtl. 1975. Analysis of atmospheric flow over a surface protrusion using the turbulence kinetic energy equation. Boundary-Layer Meteorol., 8, 401-417.

Jacobs, A. 1984. The flow around a thin closed fence. Boundary-Layer Meteorol., 28, 317-328.

Jones, W. P. and B. E. Launder. 1972. The prediction of laminarization with a two-equation model of turbulence. Int. 7. Heat Mass Transfer, 15, 301-314.

Kind, R. J. 1981. Snow drifting. In Gray, D. M. and D. H. Male, eds. Handbook of snow; principles, processes, management and use. Toronto, Pergamon Press, 338-359.

Kind, R.J. 1992. One-dimensional aeolian suspension above beds of loose particles - a new concentrationprofile equation. Atmos. Environ., 26A, 927-931.

Mellor, M. 1965. Blowing snow. CRREL Monogr., Pt. III, Section A3c.
Owen, P. R. 1964. Saltation of uniform grains in air. 7 . Fluid Mech., 20, 225-242.

Perera, M.D.A.E.S. 1981. Shelter behind two-dimensional solid and porous fences. Journal of Wind Engineering and Industrial Aerodynamics, 8, 93-104.

Pomeroy, J. W. and D. M. Gray. 1990. Saltation of snow. Water Resour. Res., 26(7), 1583-1594.

Rodi, W. 1980. Turbulence models for environmental problems. In Kollmann, W., ed. Prediction methods for turbulent flows. New York, McGraw-Hill, 258-349.

Sorbjan, Z. 1989. Structure of the atmospheric boundary layer. Englewood Cliffs, NJ, Prentice Hall.

Sutton, S. B., H. Brandt and B. R. White. 1986. Atmospheric dispersion of a heavier-than-air gas near a twodimensional obstacle. Boundary-Layer Meteorol., 35, 125153.

Tabler, R.D. 1975. Predicting profiles of snowdrifts in topographic catchments. Proc. West. Snow Conf., 43rd Annual Meeting, 87-97.

Uematsu, T., Y. Kaneda, K. Takeuchi, T. Nakata and M. Yukumi. 1989. Numerical simulation of snowdrift development. Ann. Glaciol., 13, 265-268.

Uematsu, T., T. Nakata, K. Takeuchi, Y. Arisawa and Y. Kaneda. 1991. Three-dimensional numerical simulation of snowdrift. Cold Reg. Sci. Technol., 20(1), 65-73.

The accuracy of references in the text and in this list is the responsibility of the authors, to whom queries should be addressed. 